\title{
IL DUELLO NEL RINASCIMENTO E IL RIPENSAMENTO ETICO SUL DUELLO DI FRANCESCO PATRIZI ${ }^{1}$ (SECONDA PARTE)
}

\author{
Fulvio Šuran \\ Università Juraj Dobrila di Pola \\ Dipartimento per la formazione di maestri ed educatori
}

\section{Riassunto}

Partendo dalla riflessione del Patrizi su questo delicato argomento, cercheremo di dimostrare che il duello aveva tutte le carte in regola per essere trattato con le dovute precauzioni da parte del Concilio di Trento. Esso costituiva materia quanto mai delicata e controversa che era stata oggetto di discussione da oltre tre secoli e sulla quale nel cinquantennio precedente ben cinque pontefici avevano ritenuto necessario pronunciarsi in forma solenne. Al di là del tono perentorio e ultimativo, il decreto tridentino di proibizione del duello celava un'ambiguità di fondo, in quanto rappresentava un compromesso fra posizioni fortemente contrastanti, che in quell'assise conciliare si erano espresse in modo quanto mai eloquente. Se, da una parte, il concilio di Trento sanzionò definitivamente la condanna del duello inteso come giudizio divino dall'altra, non chiuse affatto il discorso sul duello inteso come legittima difesa dell'onore e affermazione di un diritto naturale. Questione che si protrasse fino alla fine dell'ancien regimè.

È proprio nel Dialogo dell'honore, il Barignano che il Patrizi mostra di aver compreso la portata pratica delle sue affermazioni etiche riguardanti il duello e l'onore nella vita statale. Ecco perché vuole rispondere "non come persona che di duello scriva ... ma filosoficamente". In tal senso un primo problema riguarda la sua interiorità o esteriorità; un secondo problema è quello della sua positività o negatività sociale; il terzo problema investe il diritto e il dovere che questi (l'onore) rappresenta per il singolo in quanto tutelato entro i limiti e con i mezzi dello Stato o in quanto trascendente gli interessi della collettività stessa. Questo suo nuovo modo di trattare questa delicata questione eserciterà un'evidente influenza, sia nel senso positivo che negativo del termine, su tutti i numerosi politici che nei due secoli a venire discuteranno dell'onore.

Parole chiave: Concilio di Trento, Fabio Barignano, Conte Giovan Giacomo Leonardi.

1 In questa seconda parte del lavoro ci si soffermerà in particolare sul ripensamento etico dell'illustre filosofo istriano Francesco Patrizi riguardante il duello (la prima parte del lavoro è pubblicata in Studia Polensia anno I numero I, pp. 9-29). Si tratta di una discussione che il Patrizi rielabora nel suo celebre Dialogo dell'Honore, il Barignano e che ricalca le decisioni prese in merito a questa moda in auge nel Rinascimento non solo italiano, da parte del Concilio di Trento. 


\section{Il ripensamento etico sul duello di Francesco Patrizi}

\section{Francesco Patrizi tra tradizione e rinnovamento: un ripensamento etico sull'onore}

A partire dal Cinquecento divenne sempre più evidente "che quando uomini di pari valore combattevano in condizioni impari, a vincere era chi disponeva delle armi migliori"', e questo indipendentemente dall'onore cavalleresco in quanto sprovvisti dell'autorizzazione ufficiale del potere pubblico. Il tutto era dovuto non solo al decreto di condanna e alla conseguente proibizione del duello da parte dell'assise conciliare tridentina, ma altresì al fatto che gli stessi sovrani europei si rendevano sempre più conto della maggiore importanza che avevano gli elementi della meccanizzazione nelle guerre e del numero dei soldati, cioè della gens mecaniques ${ }^{3}$, coinvolti in uno scontro bellico, per cui coscienti che la virtù militare poteva affermarsi anche al di fuori del quadro nobiliare ${ }^{4}$.

$\mathrm{Fu}$ in questo periodo che i diversi principati, le signorie, le repubbliche e le monarchie dovettero, se volevano difendere la propria supremazia, abbandonare lo stile guerriero medievale, diventato ormai obsoleto, e adattarsi alle nuove modalità della guerra trasformando del tutto le proprie strategie militari, dando sempre più importanza all'uso della polvere da sparo e quindi all'artiglieria e alla fanteria in opposizione alla cavalleria. Quest'ultima, anche se era stata integrata da un imponente contingente di moschettieri ben addestrati, si era dimostrata obsoleta e iniqua di fronte al nuovo impiego della fanteria e all'uso spregiudicato dell'artiglieria, sia leggera sia pesante, il che portò alla riorganizzazione e al disciplinamento della nobiltà europea, ridimensionando il significato dell'onore individuale ancora presente nelle opere letterarie dell'Ariosto e del Tasso.

Cambiamento di valori e di virtù militari che in tutta la sua potenza rivoluzionaria era espressione del fatto che nella scelta degli strumenti militari anche la politica - a differenza di quello che ritiene $\mathrm{J}$. Keegan ${ }^{5}$ - può mostrarsi una forza altrettanto efficace della cultura dominante. Questo specialmente se sono le necessità storiche a richiedere alla politica regnante di prevalere sia sulla cultura nobiliare sia sulla stessa logica politica o militare e nel nostro caso specifico, sull'orgoglio e sull'onore mostrato dal ceto nobiliare e dalla classe militare al di fuori del campo di battaglia. Era cioè più che mai necessario rendersi conto come, in una serie di eventi, il comportamento sociale e la

\footnotetext{
2 Patrizi, F., La città felice. Dialogo dell'honore, Il Barignano. Discorso della diversità de' furori poetici. Lettura sopra il sonetto del Petrarca 'La gola, e 'l sonno, e l'ociose piume', per Giovanni Griffio, Venetia, 1553, p. 43.

Altre opere di Francesco Patrizi: Della historia diece dialoghi, ne' quali si ragiona di tutte le cose appartenenti all'historia, et allo scriverla, et all'osservarla, Venetia, 1560; Della retorica dieci dialoghi, nelli quali si favella dell'arte oratoria con ragioni repugnanti all'openione, che intorno a quella hebbero gli antichi scrittori, Venetia, 1562; Discussionum peripateticarum tomi quattuor, Basileae, 1581; Nova de universis philosophia, Ferrariae, 1591, Venetiis 1593; Della poetica, ed. critica a cura di D. Aguzzi Barbagli, 3 voll., Firenze, 1969-1971; Lettere ed opuscoli inediti, ed. critica a cura di D. Aguzzi Barbagli, Firenze, 1975; Nova de universis philosophia. Materiali per un'edizione emendata, a cura di A.L. Puliafito Bleuel, Firenze, 1993.

3 Tratto da Romano, R., Tenenti, A., Il rinascimento e la riforma (1378 - 1598), vol. IX, parte seconda, UTET, Torino, 1976, p. 173.

4 Ivi, p. 172.

5 Keegan, J., La grande storia della Guerra. Dalla preistoria ai giorni nostri, Mondadori, Milano, 1994, p. 44.
} 
valutazione morale, propri di una civiltà, mostrassero quanto il cambiamento culturale spingesse lo stesso re o signore di uno stato a delle decisioni politiche drastiche, che si opponevano agli stessi interessi della classe nobiliare che rappresentava.

In questo senso diventa più che mai necessario rendersi conto, in una serie di cambiamenti ed eventi culturali all'interno di una civiltà, di "quanto la cultura sia molto più persistente della decisione politica come causa determinante in campo militare",6, in quanto costringe la stessa 'alta' politica a seguire il suo corso se vuole sopravvivere. In questo senso il risultato del cambiamento socio-culturale è la prova tangibile che non sempre è la logica politica a condizionare l'arte militare, anzi sono proprio le sue forme culturali tradizionali che, se forti e resistenti al necessario cambiamento, possono frenare le tendenze innovatrici di ricorrere a nuove tecniche militari, questo specialmente se ne va di mezzo il ribaltamento di valori antichi e cari

Nel nostro caso specifico, si pensa al duello come prova d'onore proprio dell'aristocrazia, che si basava su principi della tradizione cavalleresca, entro la quale la difesa dell'onore, quale virtù, veniva risolta attraverso il duello dei due contendenti, il cui risultato finale era lasciato alla decisione di Dio.

C'è da dire che in definitiva lo "stile nel vestire, nell'armatura, nella fattura e nel maneggio delle armi e nel comportamento sul campo di battaglia" era un elemento centrale nel modo di vita e d'essere dell'aristocrazia non solo europea, che nell'etica cavalleresca trovava il suo punto di riferimento.

Ed è in questo contesto storico che si devono interpretare altrettanto le diverse decisioni e linee di condotta assunte in genere dai diversi governi comunali e delle signorie di quel tempo nel regolare, se non osteggiare, il duello quale forma di giudizio divino tra uguali.

Per quel che riguarda la dimensione sociale e in particolar modo quella politica e quella culturale, propria del periodo nel quale ha operato il Patrizi, c'è da dire che già nel 1541, ovvero già quattro anni prima del Concilio di Trento, il Consiglio dei Dieci della Repubblica di Venezia approvò un tipo di provvedimento legale ove si proibiva e sanzionava il duellare non solo per la Dominante, ma altresì 'nelle altre città nostre, onde procedono homicidi et altri incovenienti scandalosi'. Questo drastico cambiamento culturale della politica dominante aveva lo scopo non solo di estirpare i duelli, o almeno di sottoporli al controllo e all'autorizzazione dell'autorità statale per cui veniva tolta la residenza veneta per un periodo di dieci anni a chiunque volesse risolvere una qualsiasi questione d'onore non tramite il tribunale, bensì andando a, come si diceva in quel tempo, combattere al loco della gloria, ma altresì di infliggere un duro colpo all'arroganza del ceto nobiliare e alla tracotanza della classe militare.

La cosa divenne ancora più perentoria dopo la bolla papale emanata nel 1560 da parte di Pio IV, che preannuncia la decisa condanna, da parte della Chiesa cattolica, contro il 'detestabilis duellorum usus, fabricante Diabolo introductus', in qualità di difesa del proprio onore personale o familiare che fosse. Il che avvenne in modo decisivo nel corso della XXV sessione del Concilio di Trento, durante la quale fu comminata

$6 \quad$ Ivi, p. 44.

7 Ivi, p. 46.

$8 \quad$ Ivi, p. 47. 
la scomunica ai duellanti, ai loro consiglieri ed aiutanti nonché alle stesse autorità che avevano permesso il duello o semplicemente lo assolvevano all'interno dei loro territori. La novità di codesto concilio nei confronti di quelli che lo hanno preceduto, e quindi delle precedenti condanne in merito, sta nel fatto che la curia, resasi conto dell'impossibilità di agire direttamente sulle coscienze individuali, fa direttamente appello agli stessi reggitori dei singoli Stati. Portando in tal modo la condanna non solo ad una conseguente scomunica religiosa dei singoli individui coinvolti nel singular certamen, ma altresì trattando l'atto alla stregua di un semplice quanto incivile atto criminale per cui punibile secondo la legge.

A tale proposito riportiamo la parte del testo del decreto finale (qui tradotto in lingua italiana) che riguarda in particolar modo la questione del duello approvata da parte dei padri conciliari alla loro XXV sessione, capitolo XIX, della celeberrima assise tridentina "Noi scomunichiamo oggi e senza altra forma di processo tutti gli imperatori, re, principi, marchesi, conti e altri signori che avranno accordato qualche luogo per il duello tra Cristiani - per quelli che si saranno battuti ed i padrini volgarmente chiamati. Essi incorreranno nella pena della scomunica e nella prescrizione dei loro beni, saranno considerati infami e trattati con la stessa severità dei sacrilegi. Condannati saranno pure gli spettatori, anatema perpetuo su loro senza riguardo a privilegio di casta"."

A differenza dalle precedenti condanne riguardanti la pratica del duellare per punto d'onore - quale quella pronunciata da parte dei prelati della Chiesa cattolica durante il precedente Concilio, quello di Toledo avvenuto nel 1473, che negava la sepoltura, secondo i sacramenti, ai duellanti; nonché quella di papa Giulio II che li scomunicava in quanto pratica che andava contro quell'etica cristiana che non permetteva di arrecare morte violenta ad un altro cristiano - la novità della scomunica tridentina era dovuta all' interessante decreto omonimo che con questa chiamava in causa gli stessi reggitori dei Comuni, delle Signorie e di tutti gli Stati cristiani. Si trattava quindi di una "scomunica maggiore", in quanto implicante lo stesso diritto civile. Decreto o decisione che portò al fiorire e al dilagare di tutta una letteratura riguardante il pro e il contro della difesa dell'onore, sia individuale sia familiare, tramite il duello per punto d'onore.

Per quel che concerne la Repubblica di Venezia del tempo del Patrizi, vi era una quanto mai diffusa mania dualistica tra gli studenti, per lo più figli di ricchi commercianti e della classe nobiliare, riguardante specialmente l'Università di Padova. Studenti che nel loro orgoglio individuale si ritenevano i continuatori, se non gli eredi ideali, dei cavalieri medioevali i quali facevano (almeno questa la loro interpretazione), professione d'honore tramite il duellare in nobile tenzone.

Il tutto era dovuto agli stessi titoli o gradi universitari che, in qualità di discendenza terminologica, conferivano agli studenti le prerogative dei Cavalieri. Basti pensare che il titolo che veniva conferito in ambito universitario, e in particolar modo a chi terminava gli studi in scienze giuridiche, era appunto quello di Chevalier és lois. Si trattava del resto di uno dei titoli più ambiti tra gli studenti di tutta Europa.

A ben vedere la situazione tra i giovani di quel tempo, si tratta di una preoccupazione alquanto giustificata, sia da parte dei princìpi della Chiesa sia da parte 9 In Arcari, P. M., Il pensiero politico di Francesco Patrizi da Cherso, Zamperini e Lorenzini, Roma, 1935 , p. 148. Il terzo capitolo dell'opera è tutto dedicato a Il duello e l'onore nel clima politico del Concilio Tridentino e all'analisi dettagliata del Barignano, pp. 145-191. 
delle autorità dei diversi Stati europei e nel caso del Patrizi, dell'autorità della Repubblica di Venezia, il cui timore era rivolto a coloro che andassero a combattere al loco della sfida. Preoccupazione propria al diritto cristiano e che il Patrizi, a differenza dei giuristi del livello di un Andrea Alciato ${ }^{10}$, o dei professionisti del duello in qualità di professori d'onore, come Girolamo Muzio" ${ }^{11}$, proclama il suo ragionar filosofico sull'argomento dell'onore $^{12}$. Questione che è quanto mai chiaramente espressa dal Patrizi nella lettera dedicatoria del suo Dialogo dell'onore, il Barignano ${ }^{13}$ nella quale così parla a proposito delle varie opinioni attorno alla questione dell'onore: "Et pure di questa così grande et universal professione dell'honore, non si è ancora trovato alcuno, che principalmente n'habbia voluto scrivere. Et coloro che del Duello fino ad hora hanno scritto; a' quali più che ad altri, pare che si fosse richiesto di trattarne; presupponendolo, come cosa da tutti conosciuta, hanno fabbricato i loro discorsi, sopra fondamento incognito et oscuro; immaginando forse, che si come tutti gli huomini desiderano d'essere honorati, così dovessero anco sapere quello che si fusse l'honore; et nondimeno per prova si vede, che rarissimi sono quelli, che sappiano quale sia il vero honore"14.

Quindi, nella lettera dedicatoria al conte Giovan Francesco da Gambara nella cui casa si tenne la famosa discussione sul tema dell'onore alla quale assieme al Patrizi parteciparono presumibilmente anche i personaggi coinvolti nel suo Dialogo dell'honore, ovvero il Conte Giovan Giacomo Leonardi e lo studente patavino Fabio Barignano - sono già presenti sia $i$ temi sia $i$ motivi di cui si discuterà più avanti nel dialogo medesimo. Dall'introduzione, come si è ben visto nel testo sopra riportato e ben puntualizzato da Isabella Fedozzi, nel suo saggio, Il Barignano: Francesco Patrizi ed il dibattito sull'onore nella cultura del Cinquecento, ne consegue che:

1) Non vi è uomo che non abbia a cuore il suo onore.

2) Ne consegue che tutti aspirino ad essere considerati uomini 'da bene' e che siano disposti a vendicare ogni offesa arrecata al proprio buon nome.

3) Nonostante 'l'universal profession dell'honore', nessun scrittore si è mai occupato di definire ciò che esso veramente è. Gli autori di opere sul duello hanno dato per presupposta la natura dell'onore, con il risultato che ciascuno ha una sua opinione, reputata veridica, e fonte, invece, di infelicità perché falsa.

4) L'autore affronterà il tema dell'essenza dell'onore, riferendosi, come abbiamo detto, alle opinioni dell'ambasciatore d'Urbino ${ }^{15}$ ed alle proprie personali riflessioni.

5) Se ne occuperà non 'come persona che del duello scriva (...), ma filosoficamente' ed userà il 'modo (...) il quale il divino Platone, ne'suoi Dialoghi usa',

10 Alciato, A., Duello fatto di latino italiano a comune utilità. Tre consigli appresso della materia medesima uno de 'l detto Alciato, gl'altri de lo eccellentissimo e clarissimo giurisconsulto M. Mariano Socino, Vinegia 1552.

11 Iustinopolitano, M., Il duello. Con le risposte cavalleresche, Venetia, 1685.

12 A dir il vero, non solo il Patrizi scrisse dell'onore in senso filosofico, ma altresì Giovanni Battista Possevini che nel suo Dialogo dell'honore, Vinegia 1553, sosteneva più che mai che "il ragionar d'ingiurie s'appartiene al filosofo morale”. Da Donati, C., L'idea della nobiltà in Italia: Secoli XIV-XVIII, Laterza, Roma-Bari, 1988, p. 101.

13 Dal titolo esteso La città felice. Dialogo dell'honore, Il Barignano. Discorso della diversità de' furori poetici. Lettura sopra il sonetto del Petrarca 'La gola, e 'l sonno, e l'ociose piume', per Giovanni Griffio, Venetia, 1553.

14 Ivi, c. 20r.

15 Il Conte Giovan Giacomo Leonardi fu ambasciatore a Venezia dei duchi d'Urbino Francesco Donati I e Guidubaldo II della Rovere, dal 1531 al 1558. 
per fare 'apparire la verità' 'su una materia (...) nuova et d'altri per avanti non trattata'16. Al momento ci limitiamo a sottolineare il 'bisogno di definizione' che percorrerà la lettera e, conseguentemente, il dialogo" ${ }^{17}$.

Dalla fugace lettura della lettera dedicatoria si potrebbe concludere che il Patrizi dia un giudizio negativo su onore e duello quando, a ben guardare, si tratta di un'incomprensione di fondo riguardante la questione dell'onore, in quanto, in definitiva si tratta dell'errata presupposizione inerente l'essenza stessa dell'oggetto: l'onore. Ovvero quella di sapere che cosa l'onore sia e quali siano le dirette conseguenze comportamentali che questa mal comprensione comporta.

Per fare luce sulla questione il Patrizi, per bocca del Conte, chiede allo studente patavino, Fabio Barignano, di esprimere brevemente alcune delle definizioni più pertinenti alla natura dell'onore, il quale, a tale proposito così risponde al Conte : "Secondo quel moderno, l'honore, è uno stato incorrotto della natura. Et Aristotile dice, l'honore non esser altro che premio della virtù. Et questo medesimo dice Cicerone"18.

L'autrice Paola Maria Arcari, nell'opera Il pensiero politico di Francesco Patrizi da Cherso, ritiene che si tratti dello scritto di Fausto Longianensis (da Longiano), ovvero della sua opera Il duello del Fausto da Longiano regolato alle leggi de l'honore, e dedicato a Giacobo sesto Aragonese Appiano, ottavo Signore di Piombino ${ }^{19}$. L'Arcari è convinta che si tratti del Longiano, perché la definizione sull'onore che il Patrizi fa pronunciare a un moderno autore è simile, se non identica, a quella del summenzionato Fausto de Longiano ${ }^{20}$.

Proseguendo nella sua tesi che 'l'honore non esser altro che premio della virtù', Fabio Barignano si appoggia al principio di autorità, dato che nella sua teorizzazione prosegue con queste testuali parole: "Signor sì, perché io credo che huomini così famosi, come fu Aristotile, et Cicerone, et è ancora quell'altro moderno, non l'havrebbono detto, se non fossero state vere" ${ }_{21}$. Al quale giudizio per autorità si oppone il Conte che senza mezzi termini gli chiede di lasciare "da un canto la fama, et l'autorità di costoro. Et attendete à quello che essi dissero"'22.

Da questo momento in poi è il Conte a portare lo studente patavino a costruire, passo dopo passo, la nuova definizione di onore basandosi sulle diverse definizioni del bene quale fine dell'agire umano e della virtù in quanto "habito della nostra volontà, che sempre ci inchina ad operare bene"23. Pertanto l'onore non può che essere strettamente legato allo stesso concetto che il mondo ha degli uomini virtuosi, che sono tali in quanto agiscono nella stessa società e dalla stessa società sono conosciuti in quanto tali. Si arriva così alla filosofica definizione dell'onore del Patrizi per il quale l'onore "è un concetto buono nella mente de gli huomini generato dalla cognizione delle buone altrui

16 Ivi, cc. 20r-21r.

17 Fedozzi, I., Il Barignano: Francesco Patrizi ed il dibattito sull'onore nella cultura del Cinquecento, pp. 117-118, in (a cura di Patrizia Castelli) Francesco Patrizi, filosofo platonico nel crepuscolo del Rinascimento, Leo S. Olschki editore, Firenze, 2002, pp. 115-125.

18 Ivi, c. $23 r$.

19 Opera edita in Venezia appresso Vincenzo Valgrisi, 1552.

20 A tale proposito basti paragonare il Patrizi, op. cit., c. 23a, e il Longianensis, op. cit., p. 8.

21 Patrizi, F., op. cit., c. 23 r.

22 Ibidem.

23 Ivi, c. $34 \mathrm{r}$. 
cognizioni" ${ }^{24}$. Ma la questione riguardante l'argomento dell'onore non è ancora del tutto risolta, perché la definizione dell'onore sopra riportata non toglie ancora nulla alla legittimità di difendere l'onore tramite la pratica del duello pubblico.

Infatti, il Patrizi all'inizio del suo Dialogo dell'honore, il Barignano, a nome del Conte Lionardo fa tre richieste consecutive e sempre più mirate allo studente patavino Fabio Barignano e riguardanti certe dispute d'onore che hanno portato gli studenti dell'Università di Padova, a usar le armi per cause d'onore, in quanto giovani e turbolenti nel loro agire, per cui "tutti fanno professione d'honore"25. "Ma, che cos'è in definitiva l'onore?", si chiede giustamente il Patrizi in quanto "...stimo ancora, che la maggior parte di quelli, che ne fanno tanta professione a' nostri tempi, ne conoscano ò nulla, ò pochissima parte. Perché nel vero, di questa così gran professione, da niuno, ch'io sappia, è stato insino ad hora trattato. Et fra tante migliaia di libri che ci sono, ò pena si trova detto per trascorso quello ch'egli si sia. Et meno per ancora si è ritrovato chi abbia puntualmente diffinito l'essentia, et la natura sua" ${ }^{26}$.

Il tutto inizia con una domanda di cortesia del tipo: "Che si fa a Padova M. Fabio? com'è bello questo anno lo studio? et stanno cheti que'scolari, ò pur sono al solito rumori? ". Al doppio senso della domanda del Conte, lo studente risponde di pari misura, dando un'immagine altrettanto duplice dicendo che "Et lo studio è assai fiorito per questo anno; che si stima che arrivino presso à due mila scolari ma essi sono pur in briga, et molte nazioni stanno sull'arme ...”. Ma il problema, si chiede il Conte, riguarda la natura umana o è una questione sociale la causa dell'alterco che spinge gli scolari a stare 'sull'arme"? Infatti si domanda se "Può essere, che essi non possano fermare quei loro cervelli, et attender ò quello, ò che sono principalmente da loro padri mandati, et guardare al loro utile, et all'honesto?".

Dalla risposta che ne dà Fabio si deduce che lo stare 'sull'arme' degli scolari sia in maggior parte una questione di moda ovvero di un onore apparente, perché a tale proposito così risponde Fabio: "É quasi impossibile signor Conte che essi vivano in pace, perché tutti sono giovani et liberi, et tutti fanno profession dell'honore; senza voler patire un minimo soperchiamento da alcuno. Per il che non può essere, che non avvengano bene spesso de'scandali, tra simili persone".

Il che porta il Conte ad una prima conclusione, ovvero: "Altro ci vuole M. Fabio mio, ò fare proffesion dell'honore, che stare tutto dì in arme, andare in quadriglia, et gir dietro ò mille favole; che bisognerebbe prima sapere, quello che fosse honore, et usare ogni studio et diligenza in acquistarlo; et poi cercare in tutti i modi di mantenerlo. Quantunque io tenga, che poi che si è acquistato una fiata, non ci bisogni spendere troppa fatica in ritenerlo, che da se stesso ci sta; et ci accompagna fino alla morte; et oltre ancora ci fa immortali" ${ }^{27}$.

Dalle stesse parole del Patrizi si comprende che il Dialogo dell'honore, il Barignano, rappresenta una novità nel vastissimo mare d'opere rinascimentali incentrate sulla tematica del duello e dell'onore, la cui originalità è dovuta al fatto che l'onore

24 Ivi, cc. $36 \mathrm{v}-39 \mathrm{v}$.

25 Ivi, c. $22 \mathrm{a}$.

26 Ivi, c. 20 r.

27 Ivi, c. 22r-v. 
viene trattato da un punto di vista universale, quale quello del canone filosofico. Il che è ben visibile nella differenziazione del vero onore da quello apparente. Il primo del quale 'buon concetto' è certamente la conseguenza delle azioni veramente buone, in quanto fondate sulla virtù; dove invece il 'concetto' apparentemente buono, ovvero falso perché solo apparentemente buono, sarà la conseguenza di azioni altrettanto apparentemente buone, cioè alla moda, e che in nuce esprimono l'aspirazione verso il vero onore da conseguire per imitazione.

A tale punto c'è da chiedersi: a quali di questi due tipi di onore, quello vero o quello apparente (detto anche 'buona estimazione') fa riferimento chi si occupa del duello? A tale proposito questa è la risposta del Conte, ovvero del Patrizi, "Di niuno di questi due apertamente, ma di un terzo che è proprio de' cavalieri, et è composto da questi due"28. "Infatti il 'buon concetto' dei cavalieri è specificatamente collegato al non mancare alle regole del codice cavalleresco: il non venir meno alle norme rimanda alla 'buona estimazione', poiché non si compie alcuna azione virtuosa, ma ci si limita a non farne nessuna cattiva; mentre il non averne fatta alcuna cattiva significa essersi comunque ispirato alle regole della giustizia e del valore, che sono due virtù collegate al vero onore. Eppure 'le cause delle risse si confondono et tale va à Duello, che non sa egli medesimo a che colà lo conduca," 29 . Da cui si deduce che, chi si batte in duello per salvare il proprio onore, lo fa principalmente per ignoranza.

Dove invece, se di vero onore si tratta, è chiaro che una volta acquisito non si può perdere, poiché costruito sulla virtù del vivere onestamente. Dato che "l'habito della virtù, non si muta mai, et da lui non provengono mai operationi se non buone”, ne consegue che chi possiede virtù non può che agire con tale proposito. Sono invece da ritenersi falsi e non sono soggetti ad una 'gloria' durevole sia quella 'buona estimazione' sia quell'onore dei cavalieri che si sono macchiati di atti non virtuosi o per l'inadempienza del 'buon concetto' per cui perdono la 'stima' che gli altri hanno di $\operatorname{costoro}^{30}$.

\section{In conclusione}

Concludendo questo brevissimo saggio attorno al duello nel Rinascimento, c'è da dire che, al di là dell'originale soluzione filosofica propugnata dal Patrizi, il fenomeno della consuetudine del duello, quale istituzione universalmente diffusa da tempi antichissimi, era più che mai attuale anche in questo periodo, per cui un definitivo suo bando, quale possibile soluzione, troverà un terreno culturale ancora immaturo. Basti pensare che la maggior parte degli scrittori che si occupavano del problema, ovvero della 'scienza' duellistica, invece di preoccuparsi e di discutere sulla sua legalità, si preoccupava soprattutto di stabilire delle regole valide ed accettabili, cioè una codificazione in materia riguardante quali punti d'onore si debbano difendere con le armi. L'argomento riguardava in particolar modo il sussistere della consuetudine a battersi per difendere dei principi e dei valori incentrati sulla dignità della persona. Il

28 Ivi, c. 40r-42v.

29 Fedozzi, I., op. cit., p. 123.

30 Patrizi, F., op. cit., cc. 42v-43r. 
che doveva essere regolato secondo una data processualità nello sfidare l'antagonista (ovvero nel modo di lanciare e di ricevere le sfide).

Ed è in questo senso che il ragionamento del Patrizi si differenzia dai restanti autori rinascimentali. Nel Dialogo dell'honore si osserva una sensibilità filosofica non propria ad altri autori, in quanto egli parte da dei principi universali per approdare alla codificazione dell'onore stesso chiamato in causa dai duellanti. Grazie a questa sua originalità nel procedere, il Patrizi abbandona il piano della pura teorizzazione, per approdare alla dimensione etica e socio-politica nella sua più classica accezione filosofica; in quanto ha subito la visione della portata pratica della vita statale nelle sue affermazioni di ordine etico, che mostrano la dissoluzione politica insita nella pratica socialmente assurda del duello.

Ed è proprio partendo dal concetto dell'onore, che le discussioni insite nel Dialogo dell'honore dovevano, secondo il Patrizi, mirare immancabilmente alla definizione dell'individuo come individuo sociale, capace di virtù e in quanto tale meritevole di onore, il che portava in primo piano un problema fondamentale della vita sociale (quale massima virtù). Si capisce quindi perché il Patrizi si domandi che cosa sia l'onore, visto che molti autori - tra i quali Fausto da Langiano, Langianensis - antepongono l'onore alla vita, per cui ritengono che per ragioni d'onore il cavaliere debba battersi se vuole ritenersi un vero uomo.

Per queste ragioni il Patrizi non vuole parlare della questione dell'onore "come persona che di duello scriva (quindi quale giurista o duellante) ma filosoficamente",31, ovvero in qualità di problema filosofico. Dove, invece, le moderne trattazioni dei giuristi lo ignorano. E qui mi riferisco in modo particolare al volume, edito da Il Mulino, $I l$ Concilio di Trento e il moderno composto da autori vari e principalmente al capitolo "La proibizione del duello: Chiesa e ideologia nobiliare" 32.

Dialogo dell'honore che comunque, e proprio per la sua universalità d'intenti, eserciterà un rilevante influsso sia nel senso assertivo, ovvero positivo, sia non assertivo, o negativo, sulla maggior parte degli studiosi della politica di quel periodo storico e anche in seguito, specialmente per quel che riguarda la trattazione dell'onore, come risulta evidente già nell'opera di Lodovico Zuccolo, Discorsi dell'onore, della gloria, della reputazione e del buon concetto ${ }^{33}$, e lodato a suo tempo da Benedetto Croce in Uomini e cose della vecchia Italia ${ }^{34}$. Da non dimenticare, per quanto riguarda i tempi a noi più vicini, C. Donati e la sua già citata opera L'idea di nobiltà in Italia. Secoli $X I V-X V I I{ }^{35}$, e ancora F. Erspamer, La biblioteca di don Ferrante. Duello e onore nella cultura del Cinquecento ${ }^{36}$.

Il Patrizi, più di altri prima e dopo di lui, affronta quindi la problematica dell'onore tenendo a mente la filosofia del divino Platone, collegandola a questioni di

31 Patrizi, F., op. cit., c. 20r.

32 Angelozzi, G., La proibizione del duello: Chiesa e ideologia nobiliare, in Il Concilio di Trento e il moderno, Il Mulino, Bologna, 1996, pp. 271-308.

33 Zuccolo, L., Discorsi dell'onore, della gloria, della reputazione e del buon concetto, di Lodovico Zuccolo accademico Filiepono di Faenza, appresso Ginami, Venezia, 1623, p. 252.

34 Croce, B., Uomini e cose della vecchia Italia, Laterza, Bari, 1927.

35 Donati, C., L'idea di nobiltà in Italia. Secoli XIV-XVIII, Laterza, Roma-Bari, 1988, pp. 63-64, 97, 111.

36 Erspamer, F., La biblioteca di don Ferrante. Duello e onore nella cultura del Cinquecento, Bulzoni, Roma, 1982, pp. 96-101. 
natura socio-politica in quanto, sotto diversa forma esplicativa, riguardano il problema dei rapporti tra individuo e Stato. Problematica che il Patrizi tratterà in forma più esaustiva nelle sue opere di politica militare. Basti pensare alla "Militia romana" e ai suoi "Paralleli militari".

Sono tre - dice l'Arcari, e che io qui di seguito riprendo - i quesiti ai quali il Patrizi cerca di dare una risposta propositiva. Il primo è quello dell'interiorità o dell'esteriorità dell'onore, cioè: quale volere in sé o quale convinzione, opinione o altro che gli altri hanno di questo sottoforma di onestà? La seconda questione riguarda la positività dell'onore, cioè la sua forma attiva che si esprime sottoforma di azioni meritevoli di lode; come pure di quella passiva in qualità di bravo suddito che non manca di conformarsi alle leggi dell'onestà.

Per quel che invece riguarda la terza questione, questa si riferisce alla qualità dell'onore e al diritto e al dovere che questo rappresenta per il singolo: in qualità, si chiede il Patrizi, di quei diritti dell'individuo che lo Stato ha il dovere di tutelare all'interno dei limiti concessi alla legalità statale $\mathrm{o}$, d'altra parte, l'onore è un valore che trascende lo Stato e gli interessi della collettività stessa?

Veniamo così a trovarci di fronte a due pericolosi individualismi, il primo dei quali è di stampo cristiano, in cui l'onore riguarda prettamente l'interiorità del singolo e quindi si trova al di fuori della mediazione statale, per cui socialmente dannoso. Il secondo individualismo, da parte sua, dà all'onore quel senso di dignità personale, sottoforma di una virtù che valorizza l'individualismo, quale unità etico-morale indipendente dai valori sociali propri dello Stato.

Incentrando la discussione attorno alla questione del duello, la prima forma d'individualismo vedeva nel duello un senso di dignità, la virtù militare per eccellenza e confluente nella guerra, con elementi da debellare esaltando l'elemento dell'umiltà al di fuori di ogni limitazione terrena e quindi sociale, per ricongiungersi con Dio. Stranamente anche la seconda forma si collega a Dio, in quanto la vittoria di uno dei contendenti rappresentava un giudizio divino, quale ipotetica giustizia superiore, anche se in opposizione con la stessa giustizia statale.

C'è da osservare che nel Dialogo dell'honore il Patrizi cerca di dimostrare la negatività di ambedue le posizioni, in quanto il duello per punto d'onore del giusto è trattato come un elemento disgregatore del vivere civile, se non riportato dentro i parametri dello Stato che deve fare dell'onore un elemento di coesione sociale.

Questa coscienza civica farà sì che nel Patrizi la valorizzazione dell'onore si trasformi in ricerca di una possibile sistematizzazione e gerarchia filosofica. Ed è in un tale contesto più generico del vivere sociale che il Patrizi si avvale di Fausto Barignano per avanzare le due già nominate definizioni riguardanti l'onore: quella che fa riferimento al moderno autore, a l'altra ad Aristotele. Queste due definizioni - la prima interna e negativa, la seconda esterna e positiva - come abbiamo visto, non soddisfano il Patrizi, che si appresta a mostrarne la vacuità più che la falsità in quanto non entrano nel merito della questione dell'onore vero. Alla concezione dell'onore - propria al moderno autore - quale conservazione intatta del proprio stato di natura, il Patrizi ritiene che più dello status quo sia onorevole il moto, il progresso. Quindi alla beata ignoranza oppone il sapere sottoforma di azione, di movimento dell'anima, il che è valevole altresì per la 
stessa realtà sociale. Non solo perché le sue specificità sono soggette a progresso, ma anche perché non sono buone per se stesse in quanto possono, a seconda dei fini a cui sono indirizzate, diventare buone o cattive.

In questa sua ricerca dell'unica cosa assolutamente buona, verso la quale l'uomo deve tendere per meritare l'onore, in qualità di tensione, il Patrizi arriva al concetto di virtù quale spirito movens delle azioni buone. "Voi dite bene, che la virtù; tra le cose dell'huomo è solamente quella che sempre fa gli effetti buoni e non mai cattivi" ${ }^{\prime 37}$ dirà a tale proposito riaffermando in tal modo il carattere propositivo e positivo dell'onore. Anche se l'implicazione sociale non è del tutto evidente, in quanto intendendo l'onore quale premio della virtù, non ne rappresenta la definizione, anche se la sottintende.

Questione sociale che, nella critica alla definizione aristotelica ed esterna dell'onore, egli riprende definendo l'onore come "un concetto buono nella mente degli uominigenerata dalla cognizione delle buone altruioperationi" ${ }^{38}$. Con questa definizione dell'onore il Patrizi si avvicina, in un certo senso, ad una delle più piene formulazioni ciceroniane del cittadino, che è più compiutamente uomo perché uomo innanzi agli altri e innanzi a se stesso. Riflettendo su un'altra possibile obiezione al disprezzo filosofico dell'onore che si basa sulla mancata coincidenza fra virtù vera e virtù volgare, il Patrizi ammette che esista questa distinzione tra l'onore vero, generato da azioni veramente buone, e l'onore falso, in quanto generato da azioni solo in apparenza buone.

Il Patrizi basa l'accettazione dell'onore falso sul lato costruttivo, in quanto, secondo lui, ogni bene anche se apparente, dev'essere valutato positivamente per una sua somiglianza col vero bene, per cui "quantunque apparente è di farne gran conto" ${ }^{39}$, perché anche se non corrisponde alla vera virtù, esso rappresenta un moto verso di essa; e lo Stato ne deve tener conto. In questo senso per il Patrizi i valori etico-morali, civili e sociali includono sempre in sé la speranza del raggiungimento di quel bene comune che ha ispirato la sua Città felice, come pure tutta l'utopia rinascimentale. Ciò in quanto ad "una concezione dell'onore che minacciava di distruggere il consenso civile, il Patrizi preferiva anteporre il valore di prodotto sociale, ammettendone anche la sua intrinseca duplicità ('vero onore', buona 'estimazione'), ma salvaguardando il significato di sincera aspirazione all'elevamento verso le virtù civili che il desiderio d'onore rappresentava" 40 .

Anche se con la definizione data all'onore che, per il Patrizi, diventa una categoria dell'etica, tuttavia la sua subordinazione alla virtù, in qualità di esasperata ricerca del sommo bene e di perfezione, può di nuovo condurre al pessimismo, in quanto si tratta di una virtù obiettivamente irraggiungibile. In tal senso le posizioni filosofiche del Patrizi si differenziavano dai convincimenti generalmente accettati che si andavano radicando nella società italiana del Cinquecento, tendenti "verso una sempre più netta omogeneizzazione ideologica di segno nobiliare delle diverse classi dominanti italiane" ${ }^{41}$ secondo un modello cavalleresco totalizzante. "Del resto, il Conte Leonardi

\footnotetext{
37 Patrizi, F., op. cit, c. 34v.

38 Ivi, cc. $36 \mathrm{v}-39 \mathrm{v}$.

39 Ivi, cc. 40r-42v.

40 Fedozzi, I., op. cit., p. 124.

41 Donati, C., op. cit., p. 93.
} 
aveva osservato non solo che l'onore cavalleresco si può perdere, ma perfino che, scendendo a duello, i cavalieri non sempre hanno chiaro per quale onore combattono" "42.

Ed è per questa ragione che il Patrizi, per far fronte a questo pericolo, introduce il concetto della funzionalità dello stesso, alla quale l'autore arriva grazie alla sua preparazione filosofica platonica e alla conoscenza dell'agire politico di quello Stato di cui si sentiva suddito. In questa prospettiva, l'onore riprende la funzione di unire in un'azione meritevole non più solo il singolo individuo, ma l'intera classe dei cavalieri, "non estensibili all'intera società civile, come pretendevano i propugnatori di un modello cavalleresco totalizzante" ${ }^{43}$.

L'originalità del pensiero etico del Patrizi è inoltre da ricercare nel fatto che, in un periodo nel quale si tendeva sempre più a collegare l'onore non solo ai titoli nobiliari, ma altresì alle cariche e alla ricchezza, egli nega la complementarietà di questi con l'onore. Per il Patrizi era, infatti, quanto mai possibile altresì che "un 'huomo savio, dotto, prudente, liberale, magnanimo, giusto, forte, temperato, modesto, cortese, et virtuoso, ancor che non sia da alcun Prencipe fatto ne Governatore, ne Presidente, ne Marchese, ne Duca" ${ }^{44}$ fosse più di altri, baciato dalla dea fortuna, meritevole d'onore in quanto nobile d'animo e di spirito. Anche se rimane pur vero che, comunque, "tutti habiamo nell'animo quasi certa una credenza, che Dio non lascierebbe tanta robba ad un ricco, s'egli non fosse buono, et che un gentil'huomo, per la nobiltà del sangue, non possa essere malvagio, et che niuno, non possa salire à degnità alcuna, se non per mezzo dei mezzi propri ..." ${ }_{45}$.

42 Fedozzi, I., op. cit, p. 125.

43 Ibidem.

44 Patrizi, F., op. cit., c. 36v.

45 Ivi, cc. 40v-41r. 


\section{BIBLIOGRAFIA}

ALCIATO, A., 1552, Duello fatto di latino italiano a comune utilità. Tre consigli appresso della materia medesima uno de 'l detto Alciato, gl'altri de lo eccellentissimo e clarissimo giurisconsulto M. Mariano Socino, Vinegia.

ANGELOZZI, G., 1996, La proibizione del duello: Chiesa e ideologia nobiliare, in Il Concilio di Trento e il moderno, Il Mulino, Bologna.

ARCARI, P. M., 1935, Il pensiero politico di Francesco Patrizi da Cherso, Zamperini e Lorenzini, Roma.

BECCARIA, C., 2002, Dei delitti e delle pene, Mondadori, Milano.

BRIOIST, P., DRÉVILLON, H., et SERNA, P., 2002, Croiser le fer. Violence et culture de l'épée dans la France moderne (XVI ${ }^{-}-X V I I I^{e}$ siècles), Seyssel, Champ Vallon.

CAMPANELLA, T., PATRIZI, F., 1996, La città del sole - La città felice, Marietti, Genova.

CACCIARI, M., 1994, Geo-filosofia dell'Europa, capitolo3. Gli eroi, Adelphi, Milano.

CARDINI, F., 1992, Guerre di primavera. Studi sulla cavalleria e la tradizione cavalleresca, Le Lettere, Firenze.

CARDINI, F., 1997, L'acciar de' cavalieri. Studi sulla cavalleria nel mondo toscano e italico (secoli XII-XV), Le Lettere, Firenze.

CASTELLI, P., 2002, Francesco Patrizi, filosofo platonico nel crepuscolo del Rinascimento, Leo S. Olschki editore, Firenze.

CAVINA, M., 2005, Il sangue dell'onore. Storia del duello, Laterza, RomaBari.

CHAUCHADIS, C., 1997, La loi du duel. La code du point d'honneur dans l'Espagne des XVI ${ }^{e}-X V I I^{e}$ siècles, Presses Universitaires du Mirail, Toulouse.

CROCE, B., 1927, Uomini e cose della vecchia Italia, Laterza, Bari.

DE BORDEILLE, P., 1740, Discours sur les duels, De Brantome, Ouvres, La Haye.

DONATI, C., 1988, L'idea della nobiltà in Italia: Secoli XIV-XVIII, Laterza, Roma-Bari.

ERSPAMER, F., 1982, La biblioteca di Don Ferrante. Duello e onore nella cultura del Cinquecento, Bulzoni, Roma.

FEDOZZI, I., 2002, Il Barignano: Francesco Patrizi ed il dibattito sull'onore nella cultura del Cinquecento, pp. 117-118, in (a cura di Patrizia Castelli) Francesco Patrizi, filosofo platonico nel crepuscolo del Rinascimento, Leo S. Olschki editore, Firenze.

FLORI, J., 1999, Cavalieri e cavalleria nel Medioevo, tr. it. di M. Aboaf e S. Pico, Einaudi, Torino.

GARIN, E., 1994, Il ritorno dei filosofi antichi, Bibliopolis Editore, Napoli.

IUSTINOPOLITANO, M., 1685, Il duello. Con le risposte cavalleresche, Venetia.

KEEGAN, J., 1994, La grande storia della Guerra. Dalla preistoria ai giorni nostri, Mondadori, Milano. 
KIERNAN, G. V., 1999, Il duello. Onore e aristocrazia nella storia europea, Marsilio, Milano.

LABATUT, J.-P., 1999, Le nobiltà europee dal XV al XVIII secolo, Il Mulino, Milano.

LONGIANENSIS F., 1552, Il duello del Fausto da Longiano regolato alle leggi de l'honore, Vincenzo Valgrisi, Venezia.

PATRIZI, F., 1553, La città felice. Del medesimo. Dialogo dell'onore, il Barignano. Del medesimo. Discorso della diversità de'furori poetici. Lettura sopra il sonetto del Petrarca. La gola, el sonno, e l'ociose piume, per Giovanni Griffio, Venetia.

PRODI P., E REINHARDT W., (a cura di), 1996, Il Concilio di Trento e il moderno, Atti della XXXVIII settimana di studio (Trento, 11-15 settembre 1995), Il Mulino, Bologna.

REYFMAN, I., 1999, Ritualized Violence Russian Style. The Duel in Russian Culture and Literature, Stanford University Press, Stanford.

ROMANO, R., TENENTI, A., 1976, Il rinascimento e la riforma (1378 1598), vol. IX, parte seconda, UTET, Torino.

ZUCCOLO, L., 1623, Discorsi dell'onore, della gloria, della reputazione e del buon concetto, di Lodovico Zuccolo accademico Filiepono di Faenza, appresso Ginami, Venezia. 


\section{ETIČKO PROMIŠLJANJE DVOBOJA FRANE PETRIĆA}

Oslanjajući se na Petrićeva promišljanja o ovoj delikatnoj tematici pokušat će se dokazati da je dvoboj imao sve potrebne argumente kako bi od strane Tridentskoga sabora bio pomno tretiran. Budući da je više od tri stoljeća bio predmetom rasprava i predstavljao je delikatan i prijeporan (dvojben) predmet, o čemu svjedoči i činjenica da je čak pet vrhovnih svećenika (pape) osjetilo potrebu o njemu progovoriti. Povrh ultimativnoga tona, tridentski dekret zabrane dvoboja skrivao je, kako ćemo kasnije vidjeti slijedeći Petrićeva promišljanja, suštinsku dvosmislenost (nejasnoću, neodređenost, sumnjivost), predstavljajući zapravo kompromisno rješenje međusobno oprečnih stajališta koja su u onom crkvenom saboru vrlo jasno izložena. Ako je, s jedne strane, tridentski koncil definitivno sankcionirao (prihvatio) osudu dvoboja shvaćenoga kao božji sud (ordalia), s druge je strane ostavio otvoreno pitanje dvoboja kao opravdane (osnovane) obrane časti i izraza prirodnoga prava.

Upravo je u Dialogo dell'Honore, il Barignano Petrić dokazao da je shvatio praktične posljedice, na planu državnoga života, svojih etičkih tvrdnji u pogledu dvoboja i časti. Jasno je stoga zašto na pitanje o kakvoj je časti riječ, Petrić ne želi dati odgovor „kao osoba koja o dvoboju piše...već na filozofski način“. U tom smislu, što se časti tiče, za Petrića prvi se problem odnosi na njezinu unutrašnjost ili izvanjskost; drugi problem odnosi se na njezinu društvenu pozitivnost ili negativnost; treći problem obuhvaća pravo i dužnost koja čast predstavlja za pojedinca ako zaštićen u okrilju državnih pravila i sredstava ili ukoliko transcendentan društvenim interesima. Ponudivši novu perspektivu promišljanja o tom delikatnom pitanju Petrić je izvršio snažan utjecaj, u pozitivnom i negativnom smislu riječi, na brojne političare koji će u iduća dva stoljeća raspravljati o časti.

Ključne riječi: Tridenski sabor, Fabio Barignano, grof Giovan Giacomo Leonardi.

\section{FRANCESCO PATRIZI ETHICAL RECONSIDERATION ABOUT THE DUEL}

The debate on the sentence decree and the consequent prohibition of the duel by the Council of Trent, certainly is not one of the most important points treated at the assembly; although it had all the necessary requirements to be treated with due precautions by the members of the Council. Beyond the peremptory and final tone, the Trent decree of prohibition of the duel was hiding a basic ambiguity, as it represented a compromise between the strongly conflicting positions clearly expressed at the Council.

If on one hand the Council of Trent definitely sanctioned the condemnation of the duel as divine judgement, on the other hand it did not end the subject of the duel as self-defence of one's honour and claim of a natural right. The matter went on until the end of the ancien regime.

And it is precisely in the Dialogue of Honour, the Barignano that Patrizi shows to have understood the practical importance of his ethical statements about the duel and the honour within state life. This is why he wants to answer "not as a person writing about the duel ... but philosophically". 
In this sense the first problem regards its inwardness or outward appearance; the second problem is represented by its social positivity or negativity; the third problem regards the right and duty that it (the honour) represents for the single individual, being protected within the limits and by the means of the State or transcending the interests of the community itself.

This new way of treating this delicate subject will have an evident influence, both in the positive and negative sense of the word, on all the numerous politicians that will be discussing the matter of honour in the course of the following two centuries.

Key words: Council of Trent, Fabio Barignano, Count Giovan Giacomo Leonardi. 